Aufsatz ÖR

Prof. Dr. Markus Ludwigs und Sabine Weidermann, LL.M. Eur.

\title{
Drittwirkung der Europäischen Grundfreiheiten - Von der Divergenz zur Konvergenz?
}

\author{
Markus Ludwigs: Der Autor ist Inhaber des Lehrstuhls für \\ Öffentliches Recht und Europarecht an der Julius-Maximilians- \\ Universität Würzburg. \\ Sabine Weidermann: Die Autorin ist Wissenschaftliche Mitarbeiterin \\ am Lehrstuhl von Prof. Dr. Ludwigs.
}

Die Drittwirkung der EU-Grundfreiheiten zählt zu den immer wiederkehrenden Kernfragen des Europäischen Wirtschaftsrechts. Einen weiteren Meilenstein in der Diskussion liefert die Fra.bo-Entscheidung des EuGH vom 12. Juli 2012. Dort hat der Gerichtshof erstmals grundlegend positiv über eine direkte Bindung Privater innerhalb der Warenverkehrsfreiheit befunden. Der Beitrag ordnet diese Neuausrichtung in die bestehende Drittwirkungsdogmatik ein, klärt verbleibende Streitpunkte und gelangt zu dem Ergebnis einer gesteigerten Konvergenz aller Europäischen Grundfreiheiten.

\section{Einführung}

Die Erweiterung der Verpflichtungsadressaten für die Warenverkehrsfreiheit im aktuellen Fra.bo-Urteil ${ }^{1}$ weist in Richtung einer fortschreitenden Parallelität der Grundfreiheiten und gibt Anlass, die Drittwirkungsproblematik einer aktuellen Analyse zu unterziehen. Im Zentrum stehen die Entwicklung der Rechtsprechung des Gerichtshofs und ihre Rezeption im Schrifttum. Hierzu sind auf einer ersten Stufe Begriff und Gegenstand einer direkten Privatrechtswirkung der Grundfreiheiten zu bestimmen (II.). Sodann werden die Grundzüge der EuGH-Judikatur zu den Personenverkehrsfreiheiten und zur Dienstleistungsfreiheit skizziert (III.). In einem dritten Schritt gilt es, zunächst den vermeintlichen Gegenentwurf im Bereich der Warenverkehrsfreiheit zu beleuchten, bevor auf den mit der Rs. Fra. bo eingeleiteten Paradigmenwechsel einzugehen ist (IV.).

1 EuGH, Rs C-171/11 EuZW 2012, 797 Rn $17 \mathrm{ff}$ - Fra.bo; nachfolgend OLG Düsseldorf, Urt. v. 14. 8. 2013 - VI-2 u (Kart) 15/08, 2 u (Kart) 15/ 08 (juris); aus der Lit Ehlers JK 3/13, AEUV Art 34/2; Kloepfer/Greve DVBl 2013, 1148ff; Schmahl/Jung NVwZ 2013, 607 ff; Streinz JuS 2013, 182ff; Schweitzer EuZW 2012, $765 \mathrm{ff}$.
Abschließend soll der Fokus auf die noch offenen Fragen der Drittwirkungsdebatte gelegt werden (V.).

\section{Begriff und Gegenstand der unmittelbaren Drittwirkung}

\section{Drittwirkung im Allgemeinen}

Mit dem Begriff der Drittwirkung wird die unmittelbare oder mittelbare Bindung nichtstaatlicher (privater) Rechtssubjekte an das Unionsrecht bei der privatautonomen Ausgestaltung ihrer Horizontalverhältnisse gekennzeichnet ${ }^{2}$. Eine unmittelbare Drittwirkung liegt vor, wenn sich ein Privatrechtssubjekt direkt, d.h. ohne Zwischenschaltung des Staates, gegenüber anderen Privaten auf EU-Recht berufen $\mathrm{kann}^{3}$. Im Kontrast hierzu ist von einer mittelbaren Drittwirkung zu sprechen, wenn private Marktteilnehmer in ihren Rechtsbeziehungen zueinander zwar nicht direkt auf die unionsrechtlichen Garantien rekurrieren können, diese aber vermittels hoheitlichen Handelns zur Geltung gebracht werden. Unmittelbarer Normadressat sind dann gerade nicht die Privaten, sondern es ist der Staat, dem eine Schutzpflicht zugunsten des Einzelnen obliegt ${ }^{4}$.

Während im deutschen Verfassungsrecht eine unmittelbare Drittwirkung der Grundrechte im Grundsatz abgelehnt wird ${ }^{5}$, erweist sich die Unionsrechtsordnung prinzipiell als offener für eine direkte Privatrechtswirkung der darin enthaltenen materiellen Garantien. Im Ausgangspunkt ist freilich auch hier zu betonen, dass sich die EuGH-Judikatur bislang eher zurückhaltend im Hinblick

2 Statt vieler Körber Grundfreiheiten und Privatrecht, 2004, S 633.

$3 \mathrm{Vgl} \mathrm{z.B.}$ Canaris Bauer ua (Hrsg) Umwelt, Wirtschaft und Recht, 2002, S 29 (31ff).

4 Kingreen Die Struktur der Grundfreiheiten des EG-Rechts, 1999, S 196, »strukturelle Dreiecksbeziehung".

5 Näher Maunz/Dürig/Herdegen Grundgesetz-Kommentar, 2013, Art 1 GG Rn 59ff, auch zu den diskutierten Ausnahmen (Art 9 III 2 bzw Art 1 I GG); weitergehend noch BAG NJW 1955, 606 (607); zur Anerkennung einer mittelbaren Drittwirkung grundlegend BVerfGE 7, 198 (204ff) - Lüth. 
auf eine unmittelbare Drittwirkung der EU-Grundrechte gezeigt hat. Für ein restriktives Verständnis spricht vor allem Art. 51 I 1 GRCh. Dort werden als Grundrechtsverpflichtete allein die Organe, Einrichtungen und Stellen der EU sowie - begrenzt auf die Durchführung von Unionsrecht - die Mitgliedstaaten genannt. Vor diesem Hintergrund wird überwiegend für eine bloß mittelbare Drittwirkung der Unionsgrundrechte plädiert ${ }^{6}$. Dessen ungeachtet lassen sich in der Judikatur des EuGH aber auch vereinzelt Beispiele für die Anerkennung einer unmittelbaren Drittwirkung nachweisen. Dies gilt für den, als materielles Grundrecht $\mathrm{zu}$ qualifizierenden Grundsatz des gleichen Entgelts für Männer und Frauen aus Art. $157 \mathrm{I} \mathrm{AEUV}^{7}$ ebenso, wie für das in der Mangold-Entscheidung entwickelte Verbot der Altersdiskriminierung. Außerhalb der EUGrundrechte wird eine direkte Privatrechtswirkung des Unionsrechts bislang unstreitig für das Kartellverbot des Art. 101 AEUV und das Missbrauchsverbot des Art. 102 AEUV bejaht ${ }^{9}$. Auf grundsätzliche Ablehnung in der EuGH-Judikatur stößt dagegen die These einer Horizontalwirkung nicht ordnungsgemäß umgesetzter Richtlinien ${ }^{10}$.

Ganz im Zentrum der Drittwirkungsdogmatik steht aber die Frage einer unmittelbaren Privatrechtswirkung der EU-Grundfreiheiten. Hierzu hat sich eine ausdifferenzierte Judikatur des EuGH gebildet, die im Schrifttum kontrovers diskutiert wird. Diese Abhandlung soll zur Systematisierung beitragen und ungerklärte Fragen diskutieren.

\section{Grundlagen einer unmittelbaren Drittwirkung der Grundfreiheiten}

Im Ausgangspunkt ist festzuhalten, dass die primäre Wirkrichtung der europäischen Grundfreiheiten in der Abwehr hoheitlicher Eingriffe besteht ${ }^{11}$. Zum einen ist es

\footnotetext{
6 Statt vieler Ehlers ders (Hrsg) Europäische Grundrechte und Grundfreiheiten, 3.Aufl 2009, § 14 Rn 54; Calliess/Ruffert/Kingreen EUV/ AEUV, 4. Aufl 2011, Art 51 GRCh Rn 18 mwN.

7 Grundlegend EuGH, Rs 43/75, Slg 1976, 455 Rn 38/39, 68 - Defrenne/Sabena.

8 EuGH, Rs C-144/04, Slg 2005, I-9981 Rn 75 f, 78 - Mangold; bestätigt in EuGH, Rs C-555/07, Slg 2010, I-365 Rn 51 - Kücükdevici.

9 Statt vieler Calliess/Ruffert/Weiß (Fn 6), Art 101 Rn 13ff; Art 102 Rn 4.

10 EuGH, Rs 152/84, Slg 1986, 723 Rn 48 - Marshall; EuGH, Rs C-91/ 92, Slg 1994, I-3325 Rn 24f, 39 - Faccini Dori; Überblick bei Streinz/ Schroeder EUV/AEUV, 2. Aufl 2012, Art 288 AEUV Rn $115 \mathrm{ff}$.

11 Statt vieler Körber (Fn 2), S 79; zur Bindung der Unionsorgane jüngst EuGH, Rs C-97/09, Slg 2010, I-10465 Rn 50 - Schmelz; ausführlich Zazoff Der Unionsgesetzgeber als Adressat der Grundfreiheiten, 2011, S $70 \mathrm{ff}$ mwN.
}

typischerweise der Staat, dem eine solche legislative oder administrative Machtfülle eingeräumt wird, die es ihm erlaubt, grenzüberschreitende Wirtschaftsaktivitäten zu behindern. Zum anderen existiert nicht selten ein politischer Wille, durch die Ausgestaltung des innerstaatlichen Rechts, die im Staatsgebiet ansässigen Personen gegenüber Gebietsfremden besser zu stellen. Private haben solche marktbeschränkenden Einflussmöglichkeiten regelmäßig nicht.

Gestärkt wird die effektive Durchsetzung der Grundfreiheiten vor allem dadurch, dass der EuGH ein weites Staatsverständnis zugrundelegt. Die Bindungswirkung erstreckt sich neben öffentlichen Unternehmen (Art. 106 AEUV) ${ }^{12}$ auch auf Beliehene ${ }^{13}$ und Standesorganisationen ${ }^{14}$. Darüber hinaus wird neben förmlichen Rechtsakten auch staatliches Informationshandeln mit rein mittelbarfaktischen Auswirkungen erfass ${ }^{15}$. Der Grund für ein derart extensives Verständnis besteht darin, dass in allen genannten Fällen eine Zurechnung des Handelns zum Staat möglich ist. Hierdurch soll die Verantwortung der Mitgliedstaaten für Verstöße gegen Unionsrecht unterstrichen und eine »Flucht ins Privatrecht« ausgeschlossen werden ${ }^{16}$.

Der EuGH ist dabei allerdings nicht stehen geblieben, sondern hat seit den 1970er Jahren schrittweise auch eine unmittelbare Horizontalwirkung der Grundfreiheiten anerkannt. In der praktischen Konsequenz bedeutet dies, dass sich Private z.B. im Rahmen von Schadensersatzklagen (§ 823 II BGB) sowie bei der Geltendmachung von Unterlassungs- und Beseitigungsansprüchen (§ 1004 BGB) direkt auf eine Verletzung der Grundfreiheiten berufen können ${ }^{17}$. Dem Unionsrecht wird mithin über die klassisch-zivilrechtlichen Sanktionen zur tatsächlichen Durchsetzung verholfen ${ }^{18}$.

12 Vgl z.B. EuGH, verb Rs 188-190/80, Slg 1982, 2545 Rn 12 - Frankreich ua/Kommission.

13 Hierzu Barthel/Lepczyk JA 2008, 436 (436ff).

14 EuGH, verb Rs 266 u 267/87, Slg 1989, 1295 Rn 15f - Royal Pharmaceutical Society of Great Britain; EuGH, Rs C-292/92, Slg 1993, I6787 Rn $12 \mathrm{ff}$ - Hünermund.

15 EuGH, Rs 249/81, Slg 1982, 4005 Rn 15, 17f, 21, 29 - Buy Irish; EuGH, Rs C-325/00, Slg 2002, I-9977 Rn 14ff, 22ff - CMA-Gütesiegel.

16 Frenz Handbuch Europarecht, Bd 1, 2. Aufl 2012, Rn 326.

17 Näher z.B. Roth EWS 2013, 16 (27).

18 Reich ZfRV 2009, 148 (151). 


\section{Entwicklung der unmittelbaren Drittwirkung im Bereich der Personenverkehrsfreiheiten und der Dienstleistungsfreiheit}

Die Entwicklung der Drittwirkungslehre des EuGH hat ihren Ursprung im Bereich der Personenverkehrsfreiheiten und der Dienstleistungsfreiheit. Aus heutiger Sicht lassen sich bei systematisierender Betrachtung zwei zentrale Fallgruppen unterscheiden. Erstens plädiert der EuGH für eine unmittelbare Grundfreiheitenbindung sog. intermediärer Gewalten (1.). Innerhalb dieser Kategorie kann weiter zwischen einer Privatrechtswirkung im Innenverhältnis gegenüber den eigenen Mitgliedern (a)) und einer Verpflichtung im Außenverhältnis gegenüber Dritten (b)) differenziert werden. Zweitens hat der EuGH darüber hinaus zumindest für die Arbeitnehmerfreizügigkeit auch beim Handeln eines sonstigen (»einfachen«) Privaten eine direkte Bindung angenommen (2.).

\section{Intermediäre Gewalten}

\section{a) Unmittelbare Drittwirkung bei Handeln im Innenverhältnis}

Fall 1: Die Kläger des Ausgangsverfahrens Walrave und Koch begleiteten Radrennfahrer gegen Entgelt auf Motorrädern und boten diesen Windschatten (sog. "Steher«-Rennen). Nach den Regeln des Radsportverbands UCI für Weltmeisterschaften mussten Schrittmacher und Radrennfahrer dieselbe Staatsangehörigkeit besitzen. Die Kläger sahen hierin eine europarechtswidrige Diskriminierung. Das angerufene nationale Gericht legte dem EuGH u.a. Fragen nach der Auslegung von Art. 7, 48 und 59 I EWG-Vertrag (Art. 18, 45 und 56 AEUV) zur Vorabentscheidung vor.

Fall 2: Der belgische Profifußballer Bosman wollte nach Auslaufen seines Vertrags beim RC Lüttich zum französischen Zweitligisten US Dünkirchen wechseln. Nach den Statuten der nationalen Fußballverbände und der UEFA hatte der alte gegen den neuen Verein - unabhängig vom Auslaufen des Vertrages - Anspruch auf eine Transferentschädigung. Der RC Lüttich zweifelte an der Zahlungsfähigkeit des US Dünkirchen und verhinderte daher die Freigabeerklärung durch den nationalen Verband. Weitere Bemühungen Bosmans, einen neuen Verein zu finden, scheiterten sowohl an der Transferregelung als auch an einer Ausländerklausel in den Reglements der UEFA, die es nationalen Verbänden erlaubte, den Einsatz ausländischer Spieler zu begrenzen (»3+2-Klausel«). Bosman verklagte den RC Lüttich, den belgischen Fußballverband und die UEFA auf Schadensersatz und Unterlassung. Das zuständige Gericht befasste den EuGH in der Sache mit der Frage, ob die Transferregelungen und die
Ausländerklausel mit der Arbeitnehmerfreizügigkeit nach Art. 48 EG (Art. 45 AEUV) vereinbar seien.

Die Entscheidung in der Rs. Walrave (Fall 1) aus dem Jahr 1974 bildet den Auftakt innerhalb der ersten Fallgruppe der EuGH-Judikatur zur unmittelbaren Drittwirkung der Grundfreiheiten gegenüber intermediären Gewalten im Innenverhältnis. Der Gerichtshof stellte erstmals eine direkte Privatrechtswirkung fest, indem er das Verbot der Diskriminierung aufgrund der Staatsangehörigkeit aus den Art. 18, 45 und 56 AEUV explizit auch auf »sonstige [nichtstaatliche] Maßnahmen « anwendete, die eine »kollektive Regelung im Arbeits- und Dienstleistungsbereich enthalten $\aleph^{19}$. Zur Begründung rekurrierte er auf ein Zweifaches. Zum einen bestünde das Risiko, dass die Wirksamkeit einer Beseitigung von staatlichen Hindernissen dadurch abgeschwächt werde, „daß privatrechtliche Vereinigungen oder Einrichtungen kraft ihrer rechtlichen Autonomie derartige Hindernisse [wieder] aufrichteten $\aleph^{20}$. Zum anderen drohe bei einer Beschränkung auf staatliche Maßnahmen die Gefahr »daß das fragliche Verbot nicht einheitlich angewandt würde«, da die Arbeitsbedingungen je nach Mitgliedstaat entweder durch Gesetz, Verordnung, Vertrag oder sonstiges Rechtsgeschäft von Privatpersonen geschlossen oder vorgenommen werden ${ }^{21}$.

In der Folgezeit setzte der Gerichtshof diese Judikatur konsequent fort ${ }^{22}$, wobei zwei Aspekte Hervorhebung verdienen: Erstens erfolgte in der Rs. Van Ameyde von 1977 die Übertragung der in Walrave entwickelten Drittwirkungsgrundsätze auf die Niederlassungsfreiheit ${ }^{23}$. Zweitens nahm der Gerichtshof in der Rs. Bosman aus dem Jahr 1995 (Fall 2) eine wichtige Klarstellung dahingehend vor ${ }^{24}$, dass sich die Bindung intermediärer Gewalten vollumfänglich auf die Funktion der Grundfreiheiten als Diskriminierungs- und Beschränkungsverbote erstreckt $\mathrm{t}^{25}$. Anders als die offen nach der Staatsangehörigkeit diskriminierende »3+2-Klausel« stellte die Transferregelung eine rein beschränkende Maßnahme dar. Den EuGH hinderte dies in-

19 EuGH, Rs 36/74, Slg 1974, 1405 Rn 16/19, 20/24 - Walrave; bestätigend ua EuGH, Rs 13/76, Slg 1976, 1333 Rn 17/18 - Dona.

20 EuGH, Rs 36/74, Slg 1974, 1405 Rn 16/19 - Walrave (Hervorhebung v Verf).

21 Ibid.

22 Vgl z.B. EuGH, Rs C-176/96, Slg 2000, I-2681 Rn 35 - Lehtonen; EuGH, verb Rs C-51/96 u C-191/96, Slg 2000, I-2549 Rn 47 - Deliege; EuGH, Rs C-309/99, Slg 2002, I-1577 Rn 120 - Wouters; EuGH, Rs C325/08, Slg 2010, I-2177 Rn 30 - Olympique Lyonnais.

23 EuGH, Rs 90/76, Slg 1977, 1091 Rn 28 - Van Ameyde.

24 Ähnliche Bewertung bei Kluth AöR 122 (1997), 557 (567).

25 EuGH, Rs C-415/93, Slg 1995, I-4921 Rn 45, 83, 96, 114f - Bosman. 
des nicht daran, auch insoweit eine strenge Prüfung am Maßstab der Arbeitnehmerfreizügigkeit vorzunehmen.

\section{aa) Kritik im Schrifttum}

In der Literatur hat die Rechtsprechung des Gerichtshofs neben Zustimmung ${ }^{26}$ auch vielfältige Kritik erfahren. Im Wesentlichen werden vier Einwände formuliert ${ }^{27}$. Erstens wird auf den rein staatsgerichteten Wortlaut des AEU-Vertrags verwiesen ${ }^{28}$. Dem ist indes entgegenzuhalten, dass sich die Formulierungen in den Artikeln 34f., 45, 49, 56 und 63 AEUV bei näherer Betrachtung als neutral erwei$\operatorname{sen}^{29}$. An keiner Stelle erfolgt eine exklusive Fokussierung auf hoheitliches Handeln.

Zweitens wird in systematischer Hinsicht angeführt, dass die Rechtfertigungsgründe auf staatliche Maßnahmen zugeschnitten seien ${ }^{30}$. Die eingeschränkte Passgenauigkeit für privates Handeln bedeutet indes nicht zwingend eine Absage an eine unmittelbare Drittwirkung der Grundfreiheiten ${ }^{31}$. Zum einen können auch private Maßnahmen im Einzelfall einem Allgemeininteresse dienen ${ }^{32}$. Hieran ist insbesondere bei einem Handeln intermediärer (»staatsähnlicher«) Gewalten zu denken. Zum anderen liefern gerade die grundlegenden Entscheidungen Cassis de Dijon und Gebhard, in denen der EuGH ungeschriebene Rechtfertigungsgründe entwickelt hat ${ }^{33}$, einen Beleg für die dynamische Entwicklung der Dogmatik. Insbesondere ist kein Grund ersichtlich, weshalb sich die ungeschriebenen Rechtfertigungsgründe allein auf zwingende Erfordernisse bzw. Gründe gerade des Allgemeininteresses beziehen müssten. Selbst wenn man dies aber anders sehen wollte, würde jedenfalls die seit der Rs. Schmidberger anerkannte rechtfertigende Funktion der Unionsgrundrech-

26 Vgl etwa Hilf/Pache NJW 1996, 1169 (1176 f); Steindorff FS Lerche, 1993, S 575 (586).

27 Überblick bei Ehlers ders (Fn 6), § 7 Rn 53.

28 Körber EuR 2000, 932 (949); Burgi EWS 1999, 327 (329, 331 f). $29 \mathrm{Vgl}$ auch EuGH, Rs 36/74, Slg 1974, 1405 Rn 20/24 - Walrave; EuGH, Rs C-281/98, Slg 2000, I-4139 Rn 30 - Angonese; aus der Lit Streinz/Leible EuZW 2000, 459 (461).

30 Remmert JURA 2003, 13 (14 f); Jaensch Die unmittelbare Drittwirkung der Grundfreiheiten, 1997, S 124; Roth FS Everling, Bd 2, 1995, S 1231 (1241 f).

31 Kronberg Voraussetzungen und Grenzen der Bindung von Sportverbänden an die Europäischen Grundfreiheiten, 2011, S 63; Burgi EWS 1999, 327 (329).

32 Vgl z. B. EuGH, Rs C-415/93, Slg 1995, I-4921 Rn 106 - Bosman, wo von der »beträchtlichen sozialen Bedeutung [die Rede ist], die (...) dem Fußball in der Gemeinschaft zukommt«; aus der Lit Förster Die unmittelbare Drittwirkung der Grundfreiheiten, 2006, S 61.

33 EuGH, Rs 120/78, Slg 1979, 649 Rn 8 - Cassis de Dijon; EuGH, Rs C-55/94, Slg 1995, I-4165 Rn 37 - Gebhard. $t^{34}$ Raum für die Rechtfertigung von Eingriffen Privater in die Grundfreiheiten liefern.

Drittens wird - ebenfalls in systematischer Hinsicht darauf hingewiesen, dass der Schutz vor Handlungen Privater durch die Wettbewerbsvorschriften des AEU-Vertrages gewährleistet wird ${ }^{35}$. Zu beachten ist aber bereits, dass sich auch die Art. 101 und 102 AEUV keineswegs exklusiv an die Privaten richten. Der EuGH hat vielmehr über die Brücke des Loyalitätsgebots aus Art. 4 III EUV auch eine (mittelbare) Bindung der Mitgliedstaaten an das Kartellund Missbrauchsverbot angenommen ${ }^{36}$. Die kategorische Annahme einer alleinigen Ausrichtung des Unionsrechts auf den Staat einerseits (Grundfreiheiten) und die Privaten andererseits (EU-Kartellrecht) würde im Übrigen das umfassende Ziel der Verwirklichung des Binnenmarktes negieren und damit seine Realisierung gefährden.

Viertens wird vorgetragen, dass mit der Schutzpflichtenkonstruktion eine wirksame Alternative zur unmittelbaren Drittwirkung existiert ${ }^{37}$. Damit wird aber suggeriert, dass nur entweder eine direkte Privatrechtsbindung oder eine mittelbare Drittwirkung denkbar ist. Im Übrigen sind Konstellationen denkbar, in denen sich die Schutzpflichtenlösung als zu schwerfällig für die effektive Durchsetzung der Grundfreiheiten erweist. Hierzu kann es unter Umständen dann kommen, wenn sich eine Abhilfe nicht bereits auf behördlichem Wege, sondern erst über den Verwaltungsrechtsschutz realisieren lässt.

\section{bb) Rechtsprechung des EuGH}

Greifen die gegen eine unmittelbare Drittwirkung vorgebrachten Einwände nicht durch, ist auf die Tatsache zurückzukommen, dass private Handlungen dieselbe Gefährdungsrichtung einschlagen können wie staatliche Maßnahmen. Es kommt nicht auf den Handelnden, sondern auf die behindernden Wirkungen an. Vor diesem Hintergrund lassen der Effektivitätsgedanke (effet utile) im Allgemeinen und das Gebot der einheitlichen Anwendung des Unionsrechts im Besonderen eine unmittelbare Bindung Privater an die Grundfreiheiten im Grundsatz tragfähig erscheinen.

34 EuGH, Rs C-112/00, Slg 2003, I-5659 Rn 74 - Schmidberger. 35 Förster (Fn 32), S 83; Remmert JURA 2003, 13 (15); Körber EuR 2000, 932 (948); kritisch Kronberg (Fn 31), S 65ff; Preedy Die Bindung Privater an die europäischen Grundfreiheiten, 2005, S 106; Ganten Die Drittwirkung der Grundfreiheiten, 2000, S 82f.

36 St Rspr; grundlegend EuGH, Rs 267/86, Slg 1988, 4769 Rn 16 Van Eycke.

37 So z.B. Kingreen (Fn 4), S 195 ff; s auch V.3.b). 
Hiermit korrespondiert es, wenn auch der EuGH wiederholt auf das Vorhandensein einer rechtlichen Autonomie zum Erlass von kollektiven Regelungen als funktionales Äquivalent zu staatlichen Maßnahmen rekurriert ${ }^{38}$. Maßgeblich ist damit die Vergleichbarkeit der Gefährdungswirkung $^{39}$. Charakteristisch für den staatsähnlichen ${ }^{40}$ Auftritt intermediärer Gewalten ist der Erlass abstrakt-genereller Regelungen für eine Vielzahl von Privaten (Kriterium der Normgebung), denen diese infolge eines Über- und Unterordnungsverhältnisses unentrinnbar ausgeliefert sind (Kriterium der Unentrinnbarkeit $\left.{ }^{41}\right)$. Weitergehend wird im Schrifttum noch zum Teil auf das Merkmal eines von der intermediären Gewalt verfolgten Allgemeininteresses abgestellt $^{42}$. Hiergegen spricht aber, dass die Gefährdung der Grundfreiheiten unabhängig davon ist, welche Zwecke die intermediäre Gewalt verfolgt. Private werden regelmäßig privatnützig tätig und verfolgen dann allenfalls mittelbar Allgemeininteressen. Hierbei handelt es sich aber weniger um eine Frage der Grundfreiheitenbindung als um ein Problem der Rechtfertigung.

\section{b) Unmittelbare Drittwirkung bei Handeln im Außenverhältnis}

Fall 3: Das finnische Fährunternehmen Viking betrieb das Schiff »Rosella« unter finnischer Flagge. Die Besatzung bestand aus Mitgliedern der finnischen Gewerkschaft FSU. Diese wiederum gehörte der ITF, einer weltweiten Föderation von Gewerkschaften im Transportsektor, an. Um Lohnkosten zu sparen beschloss Viking die »Rosella" nach Estland umzuflaggen. Die FSU kritisierte dies und bat die ITF, alle angeschlossenen Gewerkschaften (u.a. auch in Estland) aufzufordern, nicht mit Viking über die Beschäftigungsbedingungen zu verhandeln. Die ITF kam dieser Bitte eines Boykottaufrufs in einem Rundschreiben an die zusammengeschlossenen Gewerkschaften nach. Daraufhin erhob Viking Klage und beantragte die ITF zu verurteilen, das Schreiben zurückzuziehen und die FSU zu verpflichten, das Umflaggungsvorhaben nicht länger zu beeinträchtigen. Dabei stützte sich Viking insbesondere auf die Niederlassungsfreiheit. Das angerufene nationale Gericht setzte das Verfahren aus und legte

38 EuGH, Rs 36/74, Slg 1974, 1405 Rn 16/19 - Walrave; EuGH, Rs C415/93, Slg 1995, I-4921 Rn 83 - Bosman; EuGH, Rs C-176/96, Slg 2000, I-2681 Rn 35 - Lehtonen; EuGH, verb Rs C-51/96 u C-191/96, Slg 2000, I-2549 Rn 47 - Deliege; EuGH, Rs C-309/99, Slg 2002, I-1577 Rn 120 - Wouters.

39 Kronberg (Fn 31), S 84.

40 Lengauer Drittwirkung von Grundfreiheiten, 2011, S $169 \mathrm{ff}, \mathrm{S} 113$, 165; Jaensch (Fn 30), S 263, 266, 286.

41 Kronberg (Fn 31), S 107; Neumann/Ogorek NZG 2008, 892 (896) 42 N. C. Ipsen Private Normenordnungen als Transnationales Recht?, 2009, S 150; Jaensch (Fn 30), S $268 \mathrm{ff}$. dem EuGH die Frage vor, ob Maßnahmen wie die von FSU und ITF mit Art. 43 EG (Art. 49 AEUV) vereinbar seien.

Nachdem der EuGH die Grundfreiheitenbindung zunächst nur »verbandsintern« für das Innenverhältnis der intermediären Gewalt zu ihren Mitgliedern (bzw. der Verbansgewalt unterworfene Personen) angenommen hatte, stellte sich in der Rs. Viking (Fall 3) die Frage, ob Gleiches auch für das Außenverhältnis gegenüber Dritten gilt. Der Gerichtshof bejahte dies sowohl für das Auftreten einer Gewerkschaft (wie der FSU) als auch eines Gewerkschaftsverbands (wie der ITF) gegenüber der Arbeitgeberseite.

Zur Begründung stützte sich der EuGH entscheidend darauf, dass die Beseitigung der Hindernisse für die Verwirklichung des Binnenmarktes gefährdet wäre, »wenn die Abschaffung der Schranken staatlichen Ursprungs durch Hindernisse neutralisiert werden könnte, die nicht dem öffentlichen Recht unterliegende Vereinigungen und Einrichtungen im Rahmen ihrer rechtlichen Autonomie setzen (...) « ${ }^{43}$. Hinter dieser Argumentation steht das bereits skizzierte Kriterium der funktionalen Äquivalenz (III.1.a)) hoheitlicher und privater Maßnahmen. Danach ist es für die Verpflichtung innerhalb der Binnenmarktfreiheiten nicht entscheidend, ob die Arbeitsbedingungen in den einzelnen Mitgliedstaaten durch Gesetz oder durch Tarifvertrag geregelt werden. In beiden Fällen ist die Gefährdungswirkung für den Binnenmarkt äquivalent und die tarifgebundenen Arbeitnehmer/Arbeitgeber sind unabhängig von hoheitlichem oder privatem Handeln gebunden (Kriterien der Normgebung und der Unentrinnbarkeit). Das Interagieren der Tarifvertragsparteien muss den Verboten der Grundfreiheiten unmittelbar unterworfen sein, da dahinter ebenso die öffentliche Gewalt hätte stehen können ${ }^{44}$. Anderenfalls würde den Mitgliedstaaten eine »Flucht in den Tarifvertrag « ermöglicht. Die unterschiedliche Regelung der Arbeitsbedingungen in den Mitgliedstaaten soll nicht durch eine Beschränkung der Grundfreiheitenbindung auf staatliche Akte zu binnenmarkthemmenden Ungleichheiten führen. Grund dafür ist erneut die effektive Durchsetzung des Unionsrechts ${ }^{45}$.

Im vorliegenden Fall 3 machte es der von der ITF unterstützte Boykottaufruf der FSU für Viking weniger attraktiv und sogar zwecklos, von der Niederlassungsfreiheit Gebrauch zu machen ${ }^{46}$. Dabei bestand zwar die Besonder-

43 EuGH, Rs C-438/05, Slg 2007, I-10779 Rn 57, s auch Rn 60 Viking (Hervorhebung v Verf).

$44 \mathrm{EuGH}$ aaO, Rn 34.

45 GA Maduro, Schlussanträge v 23. 05. 2007, Rs C-438/05, Slg 2007, I-10779 Rn 37 f - Viking. 46 EuGH, Rs C-438/05, Slg 2007, I-10779 Rn 72 - Viking. 
heit, dass nicht ein Tarifvertrag als solcher Prüfungsmaßstab der Grundfreiheiten war, sondern (Streik-) Maßnahmen mit »nicht regulativem Charakter « ${ }^{47}$. Das Handeln von FSU und ITF zielte darauf ab, Viking daran zu hindern, seine Schiffe in einem anderen EU-Mitgliedstaat registrieren zu lassen ${ }^{48}$. Hinter der Kampfmaßnahme stand aber, als übergeordneter Handlungszweck, die Ermächtigung zur Gestaltung der Verträge von abhängiger Erwerbstätigkeit ${ }^{49}$. Arbeitskampfmaßnahmen dienen der Forderung nach kollektiver Regelung der abhängigen Erwerbstätigkeit und sind demnach untrennbar mit dem Tarifvertrag verknüpft ${ }^{50}$.

Insgesamt macht die Rs. Viking deutlich, dass die Grundfreiheitenbindung intermediärer Gewalten im Lichte des effe tutile der Grundfreiheiten nicht auf das Innenverhältnis gegenüber ihren Mitgliedern beschränkt bleibt. Vielmehr wird bei (unmittelbar oder mittelbar) staatsäquivalentem Handeln auch das Auftreten im Außenverhältnis gegenüber Dritten erfasst.

\section{Sonstige (»einfache«) Private}

Fall 4: Herr Angonese, ein italienischer Staatsangehöriger deutscher Muttersprache, bewarb sich auf ein Stellenangebot einer privaten Bankgesellschaft in Bozen. In der Ausschreibung wurde verlangt, die Zweisprachigkeit (Deutsch und Italienisch) mit einem Diplom der öffentlichen Verwaltung in Bozen nachzuweisen. Die Bescheinigung wird nach einer Prüfung ausgestellt, die nur in der Provinz Bozen stattfindet. Bei den dort wohnenden Bürgern ist es üblich, sich die Bescheinigung für jeden denkbaren Fall der Arbeitssuche zu beschaffen. Für »auswärtige» Bewerber ist es aufgrund der kurzen Bewerbungsfrist schwierig, die Bescheinigung rechtzeitig zu erhalten. Da der perfekt zweisprachige Angonese die Bescheinigung nicht vorweisen konnte, wurde seine Bewerbung abgelehnt. Das von ihm angerufene italienische Gericht setzte das Verfahren aus und ersuchte den EuGH um Vorabentscheidung bezüglich der Vereinbarkeit mit der Arbeitnehmerfreizügigkeit aus Art. 39 EG (Art. 45 AEUV).

In der Rs. Angonese (Fall 4) hat der EuGH eine neue Entwicklungsstufe der Drittwirkungsdogmatik eingeleitet. Die grundlegende Innovation dieser zweiten Fallgruppe liegt in der Ausdehnung des Adressatenkreises der Arbeitnehmerfreizügigkeit auf sonstige (»einfache") Private ${ }^{51}$ in Individualverhältnissen. Vorliegend ging es gerade nicht um die Verpflichtung einer intermediären Gewalt im Rahmen von

47 So auch Roth EWS 2013, 16 (22).

48 EuGH, Rs C-438/05, Slg 2007, I-10779 Rn 73 - Viking.

$49 \mathrm{EuGH}$ aaO, Rn 65.

50 EuGH aaO, Rn 36.

51 EuGH, Rs C-281/98, Slg 2000, I-4139 Rn 36 - Angonese.
Kollektivregelungen oder -maßnahmen ${ }^{52}$. Vielmehr standen sich ein Arbeitnehmer (Angonese) und ein privater Arbeitgeber (Bankgesellschaft) gegenüber. Den Anlass bildete eine versteckte - an den Wohnsitz anknüpfende Diskriminierung aufgrund der Staatsangehörigkeit ${ }^{53}$.

Der Gerichtshof bejahte die Bindung des Arbeitgebers an die Arbeitnehmerfreizügigkeit und ging damit erstmals von einer Verpflichtung »einfacher« (d.h. nicht-staatsähnlicher) Privater im Rahmen der Grundfreiheiten aus ${ }^{54}$. Zur Begründung stützte er sich zum einen darauf, dass das in Art. 39 EG (Art. 45 AEUV) enthaltene Diskriminierungsverbot allgemein formuliert sei und sich nicht speziell an die Mitgliedstaaten richte. Zum anderen handele es sich bei Art. 39 EG um eine spezifische Anwendung des allgemeinen Diskriminierungsverbots aus Art. 12 EG (Art. 18 AEUV), das auch für Private Verbindlichkeit entfalte ${ }^{55}$.

Diese, in der Folge wiederholt bestätigte ${ }^{56}$ AngoneseRechtsprechung hat im Schrifttum neben Zustimmung auch mannigfaltige Kritik erfahren ${ }^{57}$. Als problematisch erweist sich insbesondere, dass hiermit perspektivisch der Weg in Richtung einer uneingeschränkten (»allseitigen«) unmittelbaren Drittwirkung der Grundfreiheiten vorgezeichnet sein könnte ${ }^{58}$. Das Resultat wäre eine ubiquitäre und unverhältnismäßige Einschränkung der grundrechtlich geschützten Privatautonomie ${ }^{59}$. Zudem würde die überzeugende Ausgangsthese (III.1.a)) aufgegeben, wonach die Grundfreiheiten primär auf die Abwehr staatlicher Eingriffe abzielen und privates Handeln nur bei funktionaler Äquivalenz und vergleichbarer Gefährdungswirkung erfassen.

Selbst wenn man sich dieser Kritik aber unter pauschaler - Berufung auf den effet utile nicht anschließen wollte, müsste die Annahme einer Grundfreiheiten-

$52 \mathrm{EuGH}$ aaO, Rn 34.

53 EuGH aaO, Rn 45.

54 EuGH aaO, Rn 46.

55 EuGH aaO, Rn 30, $34 \mathrm{ff}$.

56 EuGH, Rs C-94/07, Slg 2008, I-5939 Rn 45, 48 - Raccanelli; EuGH, Rs C-172/11 NZA 2012, 863 Rn 36, 51 - Erny.

57 Befürwortend Preedy (Fn 35), S 99, 102; Forsthoff EWS 2000, 389 (394, 397); kritisch Perner Grundfreiheiten, Grundrechte-Charta und Privatrecht, 2013, S 171; Bachmann AcP 2010, 424 (475 f); Pießkalla NZA 2007, 1144 (1146); Michaelis NJW 2001, 1841 (1842 f).

58 Brigola Das System der EG-Grundfreiheiten, 2004, S 91.

59 Ähnlich Röthel EuR 2001, 908 (916); ausführlich zum unionsgrundrechtlichen Schutz der Privat- und Parteiautonomie zuletzt Kroll-Ludwigs Die Rolle der Parteiautonomie im europäischen Kollisionsrecht, 2013, S $241 \mathrm{ff}$, die auf die Verankerung in einer Reihe von Einzelgrundrechten (insb Art 15 und 16 GRCh) sowie im Rahmen der als allgemeiner Rechtsgrundsatz (Art 6 III EUV) geschützten allgemeinen Handlungsfreiheit (s insoweit auch Jarass Charta der Grundrechte der EU, 2. Aufl 2013, Einl Rn $31 \mathrm{mwN}$ ) hinweist. 
bindung »einfacher" Privater jedenfalls auf den Bereich der Arbeitnehmerfreizügigkeit beschränkt bleiben ${ }^{60}$. Allein hier lässt sich nämlich typischerweise ein gewisses Machtgefälle (Über- und Unterordnungsverhältnis) mit daraus resultierender Gefährdungswirkung für die effektive Durchsetzung der Grundfreiheiten nachweisen ${ }^{61}$. Im Umkehrschluss ist eine Übertragung der Angonese-Grundsätze auf die anderen Grundfreiheiten grundsätzlich abzulehnen. Dagegen lässt sich auch nicht die vermeintliche Konvergenz der Grundfreiheiten anführen. Insoweit ist zu beachten, dass Konvergenz kein Selbstzweck ist, sondern als rechtstatsächlicher Befund aus einer normbezogenen juristischen Analyse resultiert.

\section{Gegenentwurf: Warenverkehrsfreiheit?}

Während die Rechtsprechung zur unmittelbaren Drittwirkung der Personenverkehrsfreiheiten und der Dienstleistungsfreiheit als gefestigt qualifiziert werden kann, zeichnet sich die EuGH-Judikatur zur Warenverkehrsfreiheit durch ihre Inkonsistenz aus. Im Einzelnen lassen sich drei Entwicklungsstufen nachzeichnen. Diese reichen von der apodiktischen Verneinung der unmittelbaren Horizontalwirkung über eine mittelbare Drittwirkung in Gestalt der Schutzpflichtenkonstruktion bis hin zum jüngst in der Rs. Fra.bo eingeleiteten Paradigmenwechsel in Richtung einer unmittelbaren Drittwirkung.

\section{Apodiktische Verneinung einer unmittelbaren Drittwirkung}

Im Bereich der Warenverkehrsfreiheit stellte der Gerichtshof zwar zunächst in der singulär gebliebenen Rs. Dansk Supermarked von 1981 begründungslos fest, »dass Vereinbarungen zwischen Privaten in keinem Fall von den zwingenden Bestimmungen des Vertrages über den freien Warenverkehr abweichen dürfen $\aleph^{62}$. In seiner Folgejudikatur nahm der EuGH hiervon aber Abstand und gelangte umgekehrt zur apodiktischen Verneinung einer direkten Privatrechtswirkung. Deutlich wird dies insbesondere in der Entscheidung Vlaamse Reisbureaus aus dem Jahr

60 Völlig verneinend Körber (Fn 2), S 800, 809ff.

61 Ebenso Kronberg (Fn 31), S 84f, 95, 108; Brigola (Fn 58), S 90.

62 EuGH, Rs 58/80, Slg 1981, 181 Rn 17 - Dansk Supermarked; Deutung in Richtung einer unmittelbaren Drittwirkung z.B. Pescatore FS Kutscher, 1981, S 319 (333 Fn 34).
1987. Dort hat der Gerichtshof unmissverständlich klargestellt, »[dass] sich die Artikel 30 und 34 EWG-Vertrag nur auf staatliche Maßnahmen und nicht auf Verhaltensweisen von Unternehmen beziehen (...) « ${ }^{63}$. An dieser Linie, die im Schrifttum überwiegend auf Zustimmung gestoßen ist ${ }^{64}$, hat der EuGH in nachfolgenden Entscheidungen festgehalten ${ }^{65}$.

\section{Entwicklung der Schutzpflichtendoktrin (mittelbare Drittwirkung)}

Fall 5: Auf der Brenner-Autobahn, einer zentralen Nord-SüdTransitachse, kam es zu einer Demonstration von Umweltschützern, die sich gegen den steigenden Transitverkehr wandten. Die rechtzeitig angezeigte Demonstration wurde von den österreichischen Behörden nicht untersagt und hatte eine etwa 30-stündige Blockade zur Folge. Die Behörden ließen sich von Überlegungen leiten, die mit der Achtung der Grundrechte auf Meinungsäußerungs- und Versammlungsfreiheit zusammenhingen. Herr S., ein deutscher Spediteur, klagte gegen Österreich und beanspruchte Schadensersatz dafür, dass seine LKWs während der Zeit der Demonstration nicht für Transporte von Deutschland nach Italien genutzt werden konnten. Das nationale Gericht befasste den EuGH der Sache nach mit der Frage, ob Österreich europarechtlich verpflichtet gewesen sei, die Demonstration zu untersagen.

Ungeachtet der strikten Ablehnung einer direkten Privatrechtsbindung erkannte der Gerichtshof in der Folgezeit eine mittelbare Drittwirkung an. Hierzu entwickelte er das Institut einer Schutzpflicht der Mitgliedstaaten zur Abwehr nicht-staatlicher Handelsbeschränkungen. Diese »neue Funktion « der Grundfreiheiten ${ }^{66}$ wurde bereits in der Entscheidung Spanische Erdbeeren aus dem Jahr 1997 »entdeckt $«^{67}$ und später in der Entscheidung Schmidberger (Fall 5) von 2003 bestätigt. In beiden Fällen bestand trotz der fehlenden unmittelbaren Zurechenbarkeit der privaten Einfuhrbeschränkungen und Handelsbehinderun-

63 EuGH, Rs 311/85, Slg 1987, 3801 Rn 30 - Vlaamse Reisebureaus; in diesem Sinne bereits vorher EuGH, verb Rs 177 u 178/82, Slg 1984, 1797 Rn 11f, 14 - Van de Haar; EuGH, Rs 251/83, Slg 1984, 4177 Rn 20 - Haug-Adrion.

64 Vgl z.B. Körber EuR 2000, 932 (949 f); Möschel NJW 1994, 781 (782); aA Moench, NJW 1982, 2689 (2690).

65 EuGH, Rs 65/86, Slg 1988, 5249 Rn 11f - Bayer; EuGH, Rs C-159/ 00, Slg 2002, I-5031 Rn 74 - Sapod Audic.

66 Szczekalla DVBl 1998, 219 (219).

67 EuGH, Rs C-265/95, Slg 1997, I-6959 Rn 30 ff - Spanische Erdbeeren; zuvor bereits aus der Lit Jarass EuR 1995, 202 (211); Schaefer Die unmittelbare Wirkung des Verbots der nichttarifären Handelshemmnisse (Art 30 EWGV) in den Rechtsbeziehungen zwischen Privaten, 1987, S $264 \mathrm{f}$. 
gen ${ }^{68}$ zur öffentlichen Hand eine objektive Gefährdung der Warenverkehrsfreiheit. Vor diesem Hintergrund leitete der EuGH mittelbar über die Garantie der Warenverkehrsfreiheit und Art. 10 EG (Art. 4 III 2 EUV) eine staatliche Schutzpflicht $\mathrm{ab}^{69}$. Danach muss der Mitgliedstaat alle erforderlichen und geeigneten Maßnahmen ergreifen, damit der freie Warenverkehr nicht durch Handlungen von Privatpersonen beeinträchtigt wird ${ }^{70}$. Innerhalb dessen kommt den zuständigen Stellen zwar ein weites Ermessen $z^{71}$. Die Mitgliedstaaten unterliegen aber immerhin einer Beachtung von Ermessensgrenzen, indem sie im Sinne eines Untermaßverbots $^{72}$ verpflichtet sind, ausreichend gegen Verletzungen durch Private vorzugehen ${ }^{73}$.

In der Literatur wurde die Anerkennung der Schutzpflichtenkonstruktion zum Teil als endgültige Absage an eine unmittelbare Drittwirkung der Warenverkehrsfreiheit interpretiert ${ }^{74}$. Dabei blieb indes unberücksichtigt, dass der Gerichtshof hierzu keine klare Aussage gemacht hatte und nach den Vorlagefragen auch nicht machen musste (V.3.b)). Umgekehrt könnte sogar argumentiert werden, dass der EuGH implizit die Relevanz privater Beeinträchtigungen für die Grundfreiheiten deutlich gemacht hat ${ }^{75}$. Vor dem Hintergrund des Binnenmarktziels erscheint es jedenfalls nicht widersprüchlich, wenn er jüngst in der Fra.bo-Entscheidung noch einen Schritt weiter in Richtung unmittelbarer Drittwirkung gegangen ist.

\section{Die Fra.bo-Entscheidung: Paradigmenwechsel oder Akzentverschiebung?}

Fall 6: Die in Italien ansässige Gesellschaft Fra.bo stellt sog. Kupferfittings (Verbindungsstücke) für Gas- und Wasserleitun-

68 Preedy (Fn 35), S 39, »tatsächliches Handeln Privater«; Röthel EuR 2001, 908 (913), »faktische Binnenmarktbeeinträchtigungen«. 69 EuGH, Rs C-265/95, Slg 1997, I-6959 Rn 32, 39, 42, 66 - Spanische Erdbeeren; EuGH, Rs C-112/00, Slg 2003, I-5659 Rn 22, 59 f, 64 Schmidberger.

70 EuGH, Rs C-265/95, Slg 1997, I-6959 Rn 32 f, 39, 52, 56 - Spanische Erdbeeren; EuGH, Rs C-112/00, Slg 2003, I-5659 Rn 59 f - Schmidberger.

71 EuGH, Rs C-265/95, Slg 1997, I-6959 Rn 33 - Spanische Erdbeeren; EuGH, Rs C-112/00, Slg 2003, I-5659 Rn 82 - Schmidberger.

72 Grundlegend zum Untermaßverbot im nationalen Recht Canaris AcP 184 (1984), 201 (228); für eine Übertragbarkeit auf die Dogmatik der EU-Grundfreiheiten auch Frenz (Fn 16), Rn 733.

73 Canaris (Fn 3), S 52ff; Meurer EWS 1998, 196 (200).

74 So etwa Burgi EWS 1999, 327 (330 f); Hirsch ZEuS 1999, 503 (508). 75 EuGH, Rs C-265/95, Slg 1997, I-6959 Rn 31 - Spanische Erdbeeren; EuGH, Rs C-112/00, Slg 2003, I-5659 Rn 58 - Schmidberger; ähnliche Deutung auch bei Suerbaum EuR 2003, 390 (394 f). gen her und vertreibt diese. Dafür beantragte sie in Deutschland die Zertifizierung der Verbindungsstücke beim DVGW, einem gemeinnützigen privatrechtlichen Verein ohne Gewinnerzielungsabsicht. Die vom DVGW erarbeiteten Normen werden durch die Verordnung über Allgemeine Bedingungen für die Versorgung mit Wasser (AVBWasserV) explizit in Bezug genommen. Danach wird im Falle einer Zertifizierung durch den DVGW die Konformität mit dem nationalen Recht vermutet. Fra.bo hatte ein entsprechendes Zertifikat zunächst befristet erhalten. Im Rahmen einer Normänderung und des daraus resultierenden obligatorischen Nachprüfungsverfahrens stellte Fra.bo jedoch keinen Antrag auf Zusatzzertifizierung. Daraufhin wurde ihr das Zertifikat wieder entzogen bzw. nicht verlängert. Fra.bo erhob Klage auf Erteilung des Zertifikats mit der Begründung eines Verstoßes gegen EU-Recht. Das angerufene nationale Gericht ersuchte den EuGH um Vorabentscheidung im Hinblick auf die Auslegung der Art. 28, 81 und 86 II EG (Art. 34, 101 und 106 II AEUV).

In seinem Fra.bo-Urteil vom 12. Juli 2012 hält der Gerichtshof auf einer ersten Stufe fest, dass es sich beim DVGW um eine private Einrichtung handelt, deren Tätigkeit nicht dem Staat zugerechnet werden kann, da weder eine staatliche Finanzierung, noch ein maßgeblicher hoheitlicher Einfluss vorliegt ${ }^{76}$. Vor diesem Hintergrund stellt sich in einem zweiten Schritt die Frage, »ob die Tätigkeit einer privatrechtlichen Einrichtung wie des DVGW (...) ebenso wie staatliche Maßnahmen Behinderungen des freien Warenverkehrs zur Folge hat « ${ }^{77}$.

Der Gerichtshof bejaht dies und stützt sich insoweit auf dreierlei Erwägungen: Erstens habe der deutsche Gesetzgeber in $\S 12$ IV AVBWasserV die Vermutung aufgestellt, dass Erzeugnisse, die vom DVGW zertifiziert werden, dem nationalen Recht entsprechen ${ }^{78}$. Zweitens sei der DVGW »die einzige Einrichtung, die die im Ausgangsverfahren in Rede stehenden Kupferfittings im Sinne von $\S 12$ IV AVBWasserV zertifizieren kann (...) « $\ll^{79}$. Diese faktische Monopolstellung des DVGW wird nach Ansicht des Gerichtshofs auch nicht durch die Alternative eines Sachverständigenverfahrens abgemildert. Denn die einschlägige Methode sei für die Wirtschaftsteilnehmer aus administrativen und finanziellen Gründen »(...) wenig oder gar nicht praktikabel (...) « ${ }^{80}$. Drittens unterstreicht der Gerichtshof, dass »(...) nach Ansicht des vorlegenden Gerichts in der Praxis das Fehlen einer Zertifizierung durch den DVGW den Vertrieb der betreffenden Erzeugnisse auf dem deutschen Markt erheblich [erschwert]«. Eine Einrichtung wie der DVGW verfüge daher »(...) in Wirklichkeit über die

76 EuGH, Rs C-171/11 EuZW 2012, 797 Rn 24 - Fra.bo. 77 EuGH aaO, Rn 26 (Hervorhebungen v Verf).

$78 \mathrm{EuGH}$ aaO, Rn 17, 27, 32.

$79 \mathrm{EuGH}$ aaO, Rn 17, 27, 29, 32.

80 EuGH aaO, Rn 29. 
Befugnis (...), den Zugang (...) [der] Kupferfittings zum deutschen Markt zu regeln « ${ }^{81}$.

Hinter diesem schlüssigen argumentativen Dreiklang aus gesetzlicher Konformitätsvermutung, Monopolstellung und Marktzugangsbeschränkung steht letztlich das, in Rn. 26 der Urteilsgründe zum Ausdruck kommende, grundlegende Kriterium der funktionalen Äquivalenz. Dieses bildet den übergeordneten Prüfungsrahmen für die vorstehenden drei Erwägungen. Im Einklang mit der Judikatur zur unmittelbaren Drittwirkung im Bereich der Personenverkehrsfreiheiten und der Dienstleistungsfreiheit kommt damit auch innerhalb der Warenverkehrsfreiheit das Merkmal der rechtlichen Autonomie als funktionales Äquivalent zu staatlichen Maßnahmen zum Ausdruck (Kriterien der Normgebung [hier in Gestalt der Erstellung von technischen Normen $]^{82}$ und der Unentrinnbarkeit). Im Sinne der effektiven Durchsetzung der Grundfreiheiten können im Rahmen eines Tests des hypothetischen Staatshandelns auch nicht-staatliche Beschränkungen der Binnenmarktfreiheiten erfasst und einer Grundfreiheitenprüfung unterzogen werden.

Bemerkenswert erscheint es freilich, dass sich der Gerichtshof an keiner Stelle mit seiner überkommenen Judikatur zur unmittelbaren Drittwirkung im Bereich der Warenverkehrsfreiheit (IV.1.) auseinandersetzt. Kritiker nehmen diese Tatsache zum Anlass, die Fra.bo-Entscheidung abzulehnen bzw. als besonderen Einzelfall zu deuten, dem keine allgemeine Aussage zur Drittwirkung für intermediäre Gewalten im Bereich der Warenverkehrsfreiheit zu entnehmen ist ${ }^{83}$.

Zur Begründung werden drei Argumente vorgetragen. Diese umfassen neben der besonderen Struktur der Warenverkehrsfreiheit und der vorrangigen Anwendung der Wettbewerbsvorschriften auch den Hinweis auf eine Verantwortlichkeit des Staates aufgrund des Vorliegens einer »staatlichen Verweisungsnorm ${ }^{84}$. Keiner der drei Einwände greift indes durch. Was zunächst die vermeintliche Eigenart der Warenverkehrsfreiheit als Produktfreiheit angeht, so ist auf den Vergleich mit der Dienstleistungsfreiheit zu verweisen, die ebenfalls durch ihren produktbezo-

81 EuGH aaO, Rn 22, $30 \mathrm{fiVm} .26$.

82 GA Trstenjak, Schlussanträge v 28. 03. 2012, Rs C-171/11 BeckRs 2012, 80688 Rn 43ff - Fra.bo, »De-facto-Regelsetzungskompetenz« (Rn 45 - Hervorhebung im Original); s auch III.1.

83 In diese Richtung Schmahl/Jung NVwZ 2013, 607 (610); Schweitzer EuZW 2012, 765 (767 f).

84 Schmahl/Jung NVwZ 2013, 607 (611f); Schweitzer EuZW 2012, 765 (768). genen Charakter geprägt ist ${ }^{85}$ und für die eine unmittelbare Drittwirkung in ständiger Rechtsprechung anerkannt wird $^{86}$. In systematischer Hinsicht erscheint daher die Judikatur zur direkten Privatrechtsbindung dieser Freiheit auch auf die Warenverkehrsfreiheit übertragbar.

Ebenso wenig überzeugen kann der Einwand des Vorrangs der unionsrechtlichen Wettbewerbsvorschriften. Dazu ist zum einen anzumerken, dass der EuGH im Fra.boUrteil die zweite Vorlagefrage (Wettbewerbsvorschriften) nur im Falle einer Verneinung der ersten (unmittelbare Drittwirkung) geprüft hätte ${ }^{87}$. Zum anderen ist bereits unter III.1.a)aa) ausgeführt worden, dass die Wettbewerbsvorschriften des AEU-Vertrages und die Europäischen Grundfreiheiten nicht in einem kategorischen Alternativverhältnis stehen.

Was schließlich die "staatliche Verweisung« betrifft, so hat der EuGH in einem vorgeschalteten Schritt festgestellt, dass die private Tätigkeit des DVGW nicht hoheitlich zugeordnet werden kann (s.o.). Der Gerichtshof hat demnach eine, dem »Staat zurechenbare Konformitätsvermutung " für einen maßgebenden staatlichen Einfluss auf den DVGW nicht ausreichen lassen ${ }^{88}$. Für dieses Ergebnis spricht die faktische Monopolstellung des DVGW, die nicht zwingend von der "staatlichen Verweisung" abhängig ist ${ }^{89}$, da die gesetzliche Konformitätsvermutung nach der AVBWasserV grundsätzlich für das Zeichen jedes akkreditierten Branchenzertifizierers (nach a.F.: anerkannte Prüfstelle) gilt. Zudem steht die Marktzugangsbeschränkung nicht in unmittelbarem Zusammenhang mit der »staatlich zurechenbaren Konformitätsvermutung«, denn die Zertifizierung liegt allein in der Verantwortung des DVGW. Eine derartige Verweisung reicht demnach nicht aus, um den Staat wegen einer Verletzung der Warenverkehrsfreiheit zu verpflichten. Würde der Gerichtshof dann nicht wie im vorliegenden Fall Fra.bo eine unmittelbare Drittwirkung annehmen, könnte objektiven Beeinträchtigungen der Grundfreiheiten nicht wirksam begegnet werden. Das Vorgehen des EuGH lässt sich konsequent auf die tatsächliche

85 So auch Schwarze/Holoubek EU-Kommentar, 3. Aufl 2012, Art 56, 57 AEUV Rn 2, $7 \mathrm{f} \mathrm{mwN;} \mathrm{Pache} \mathrm{Ehlers} \mathrm{(Fn} \mathrm{6),} \mathrm{§} 11$ Rn 3; ferner Kingreen von Bogdandy/Bast (Hrsg) Europäisches Verfassungsrecht, 2. Aufl 2009, 705 (746).

86 Vgl die Nachweise in Fn 38.

87 EuGH, Rs C-171/11 EuZW 2012, 797 Rn 16 - Fra.bo.

88 Dazu Kloepfer/Greve DVBl 2013, 1148 (1152); Streinz JuS 2013, 182 (183); s auch EuGH, Rs C-171/11 EuZW 2012, 797 Rn 24 - Fra.bo, wo der EuGH es sogar als »unstreitig « bezeichnet, dass Deutschland »keinen maßgebenden Einfluss auf die Normungs- und Zertifizierungstätigkeiten des DVGW ausübt«.

89 AA Schmahl/Jung NVwZ 2013, 607 (612); Schweitzer EuZW 2012, 765 (767f). 
Gefährdungswirkung privater Marktzugangsbeschränkungen zurückführen, denen im Lichte des Kriteriums der funktionalen Äquivalenz über das Instrument der unmittelbaren Drittwirkung abgeholfen werden kann.

Vor diesem Hintergrund spricht mehr dafür, die Fra. bo-Entscheidung nicht nur als bloße Akzentverschiebung, sondern als grundlegenden Paradigmenwechsel innerhalb der EuGH-Judikatur zur Warenverkehrsfreiheit zu deuten. In diesem Sinne handelt es sich zugleich um einen bedeutsamen Schritt in Richtung einer Konvergenz der Grundfreiheiten.

\section{Offene Fragen}

Die bisherigen Ausführungen waren einer kritischen Analyse der vorliegenden EuGH-Judikatur zur (unmittelbaren) Drittwirkung der Grundfreiheiten gewidmet. Ungeachtet der dabei festgestellten Vielzahl gesicherter Erkenntnisse bestehen noch offene Fragen, die nachfolgend einer Klärung zugeführt werden sollen.

\section{Unmittelbare Drittwirkung bei rein beschränkenden Maßnahmen sonstiger (»einfacher«) Privater?}

In den ersten Entscheidungen des EuGH zur unmittelbaren Drittwirkung standen ausschließlich diskriminierende Maßnahmen Privater auf dem Prüfstand ${ }^{90}$. In der Entscheidung Bosman stellte der Gerichtshof dann - wie gezeigt (III.1.a)) - klar, dass eine unmittelbare Drittwirkung auch für rein beschränkende Maßnahmen gilt, die »unabhängig von der Staatsangehörigkeit (...) Anwendung finden « ${ }^{91}$. Noch offen ist in Anbetracht des Sonderfalls Angonese dagegen, ob die Grundfreiheiten auch gegenüber »einfachen « Privaten in ihrer Funktion als Diskriminierungs- und Beschränkungsverbote zum Tragen kommen ${ }^{92}$. Im Schrifttum wird die Judikatur des EuGH z.T. dahingehend gedeutet, dass sonstige (»einfache«) Private im Grundsatz »nur« bei Diskriminierungen und intermediäre Gewalten auch bei Beschränkungen Adressaten der Grundfreiheiten sein kön-

90 EuGH, Rs 36/74, Slg 1974, 1405 Rn 16/19 - Walrave.

91 EuGH, Rs C-415/93, Slg 1995, I-4921 Rn 96 - Bosman; bestätigt in EuGH, verb Rs C-51/96 u C-191/96, Slg 2000, I-2549 Rn 64 - Deliege.

92 EuGH, Rs C-281/98, Slg 2000, I-4139 Rn 36ff - Angonese, wo herausgearbeitet wurde, dass die Arbeitnehmerfreizügigkeit vorliegend als Diskriminierungsverbot zur Anwendung gelangte. nen ${ }^{93}$. Hiergegen ist aber zum einen einzuwenden, dass sich der Gerichtshof zur Problematik bislang überhaupt noch nicht äußern musste. Zum anderen hängt das Gefährdungspotential für den Binnenmarkt nicht entscheidend von der - ohnehin vielfach nicht trennscharf möglichen ${ }^{94}-\mathrm{Ab}$ grenzung zwischen diskriminierenden und rein beschränkenden Maßnahmen $a b^{95}$. Berücksichtigung kann diese Unterscheidung somit allenfallsim Rahmen der Abwägung auf der Rechtfertigungsebene finden. Wird daher eine unmittelbare Drittwirkung von sonstigen (»einfachen«) Privaten bejaht, muss sich diese bei konsequenter Betrachtung auch auf reine Beschränkungen erstrecken.

\section{Unmittelbare Drittwirkung im Rahmen der Kapitalverkehrsfreiheit?}

Eine zweite Lücke in der Drittwirkungs-Judikatur des EuGH besteht im Fehlen einschlägiger Entscheidungen zur Kapitalverkehrsfreiheit ${ }^{96}$. Von diesem rein faktischen Befund kann freilich - entgegen vereinzelter Stimmen im Schrifttum ${ }^{97}$ - nicht auf eine Verneinung der direkten Privatrechtswirkung von Art. 63 AEUV geschlossen werden. Nur im Ergebnis kann es ferner überzeugen, wenn im jüngeren Schrifttum aus der Fra.bo-Entscheidung des EuGH eine unmittelbare Drittwirkung auch für die Kapitalverkehrsfreiheit abgeleitet wird ${ }^{98}$. Die insoweit erfolgende Argumentation mit dem produktbezogenen Charakter beider Grundfreiheiten erfasst das Wesen der Kapitalverkehrsfreiheit nur partiell. Richtigerweise handelt es sich bei der Kapitalverkehrsfreiheit um eine »gemischte« Freiheit. Diese setzt sich aus Elementen der Produktverkehrsfreiheiten zusammen. Exemplarisch hierfür sind die rendite- und spekulationsorientierten Portfolioinvestitionen zu nennen, bei denen das »Finanzprodukt« im Mittelpunkt steh $^{99}$. Darüber hinaus weist die Kapitalverkehrsfreiheit aber auch Verwandtschaft mit der Niederlassungsfreiheit als Personenverkehrsfreiheit auf. Dies verdeutlicht der Blick auf den grundfreiheitlichen Schutz von Direktinvestitionen, die dadurch gekennzeichnet sind, dass der Investor mit seiner Vermögensbeteiligung kontrollierenden Ein-

93 Preedy (Fn 35), S 55, 60f; gegen jede Drittwirkung des Beschränkungsverbots Forsthoff EWS 2000, 389 (393).

$94 \mathrm{Vgl} \mathrm{EuGH}$, Rs C-281/98, Slg 2000, I-4139 Rn 41 - Angonese.

95 So im Ergebnis auch Brigola (Fn 58), S 91.

96 Verse ZIP 2008, 1754 (1759); Wagner ÖJZ 2007, 634 (638).

97 Körber EuR 2000, 932 (950).

98 Schmahl/Jung NVwZ 2013, 607 (610).

99 EuGH, verb Rs C-282/04 und C-283/04, Slg 2006, I-9141 Rn 19 Kommission/Niederlande. 
fluss auf das Unternehmen ausüben möchte ${ }^{100}$. Hier sind im Grundsatz sowohl die Niederlassungs- als auch die Kapitalverkehrsfreiheit einschlägig ${ }^{101}$ wobei die Auflösung des Konkurrenzverhältnisses zu den umstrittensten Fragen der Grundfreiheitendogmatik zählt ${ }^{102}$.

Auch wenn es sich bei der Kapitalverkehrsfreiheit mithin nicht um eine reine Produktverkehrsfreiheit handelt, ändert dies nichts am Ergebnis der Anerkennung einer unmittelbaren Drittwirkung auch im Rahmen von Art. 63 AEUV. In Rechnung zu stellen ist insoweit, dass eine unmittelbare Drittwirkung sowohl der Produktverkehrsfreiheiten als auch der Personenverkehrsfreiheiten seit der Fra.bo-Entscheidung durchgängig der EuGH-Judikatur entspricht. Damit ist kein Grund ersichtlich, weshalb die Drittwirkungsdoktrin vor der Kapitalverkehrsfreiheit als "gemischter« Freiheit Halt machen sollte ${ }^{103}$.

\section{Abgrenzung von mittelbarer und unmittelbarer Drittwirkung}

Zwei weitere umstrittene Fragen ergeben sich mit Blick auf die Abgrenzung von mittelbarer und unmittelbarer Drittwirkung. Klärungsbedürftig ist hier zum einen, inwieweit die Schutzpflichtenkonstruktion als Spielart der mittelbaren Drittwirkung auf die anderen Grundfreiheiten übertragbar ist. Zum anderen ist der Frage des Verhältnisses von Schutzpflichten einerseits und unmittelbarer Privatrechtswirkung andererseits nachzugehen.

\section{a) Erstreckung der Schutzpflichtenkonstruktion auf alle Grundfreiheiten?}

Was zunächst die Erstreckung der Schutzpflichtenkonstruktion angeht, so spricht hierfür entscheidend, dass auch bei den anderen Grundfreiheiten Fallgestaltungen denkbar sind, in denen private Beeinträchtigungen des Binnenmarktes effektiv nur im Wege eines hoheitlichen Handelns in Erfüllung einer staatlichen Schutzpflicht be-

100 Ibid.

101 Vgl z.B. EuGH, Rs C-367/98, Slg 2002, I-4731 Rn 38, 56 - Kommission/Portugal; aus der Lit Schwarze/Glaesner (Fn 85), Art 63 AEUV Rn 11f.

102 Statt vieler Streinz/Sedlaczek/Züger (Fn 10), Art 63 AEUV Rn $31 \mathrm{ff}$.

103 So im Ergebnis auch GA Trstenjak, Schlussanträge v 28. 03. 2012 Rs C-171/11 BeckRs 2012, 80688 Rn 44 - Fra.bo. seitigt werden können ${ }^{104}$. Exemplarisch sind insoweit die Besetzung von Niederlassungen ausländischer Unternehmen oder gewalttätige Ausschreitungen gegen Arbeitnehmer, Dienstleister oder Finanzinstitute aus anderen Mitgliedstaaten $\mathrm{zu}$ nennen ${ }^{105}$. Nicht zuletzt mit der Fra.boEntscheidung hat der EuGH im Übrigen bestätigt, dass die Warenverkehrsfreiheit keine dogmatische »Sonderstellung " im System der Grundfreiheiten aufweist. Auch in struktureller Hinsicht sind daher keine Bedenken gegen eine Übertragung der Schutzpflichtendoktrin auf die anderen Binnenmarktfreiheiten erkennbar.

\section{b) Alternativität oder Kumulation?}

Was im Weiteren das Verhältnis von mittelbarer und unmittelbarer Drittwirkung der Grundfreiheiten betrifft, so sind im Wesentlichen zwei Ansichten denkbar. Der erste Ansatz ist dadurch charakterisiert, dass bei jeder einzelnen Grundfreiheit ein striktes Alternativitätsverhältnis besteht. Dies würde bedeuten, dass auf einer abstrakten Ebene stets nur entweder eine unmittelbare oder eine mittelbare Drittwirkung denkbar wäre ${ }^{106}$. Die Anerkennung einer Schutzpflicht des Staates für alle Grundfreiheiten (V.III.a)) schließt es indes nach dem vorzugswürdigen Gegenmodell nicht aus, zu dieser auch kumulativ eine direkte Privatrechtsbindung treten $\mathrm{zu}$ lassen ${ }^{107}$.

Hierfür lässt sich erstens die EuGH-Rechtsprechung in den Rs. Spanische Erdbeeren und Schmidberger anführen. Dort stand nämlich gerade kein »Entweder-Oder-Verhältnis« im Raum. Die Ausführungen des Gerichtshofs enthalten daher auch keinerlei Anhaltspunkte im Sinne einer Ablehnung der Lehre von der direkten Privatrechtsbindung $^{108}$. Der Grund dafür ist, dass beide Fälle allein auf das staatliche Handeln bzw. Unterlassen eines Mitgliedstaats

104 Vgl EuGH, Rs C-438/05, Slg 2007, I-10779 Rn 61f - Viking; aus der Lit Kronberg (Fn 31), S 106.

105 Meurer EWS 1998, 196 (202).

$106 \mathrm{Vgl}$ für eine ausschließlich mittelbare Drittwirkung Canaris (Fn 3), S 49ff; Körber EuR 2000, 932 (951); Streinz/Leible EuZW 2000, 459 (465ff); Burgi EWS 1999, 327 (330 f).

107 In diese Richtung auch Kronberg (Fn 31), S 105; Brigola (Fn 58), S 90; Vieweg/Röthel ZHR 2002, 6 (20); zu weitgehend aber Roth (EWS 2013, 16 [24f]) und Perner ([Fn 57], S 170), die von einer logisch zwingenden Verknüpfung zwischen staatlicher Schutzpflicht und direkter Privatrechtsbindung ausgehen. Hiergegen spricht, dass zwischen der materiellen Beschränkung der Grundfreiheit einerseits und der formalen Stellung als Verpflichtungsadressat andererseits zu unterscheiden ist.

108 EuGH, Rs C-265/95, Slg 1997, I-6959 Rn 32 - Spanische Erdbeeren; EuGH, Rs C-112/00, Slg 2003, I-5659 Rn 59 - Schmidberger; aus der Lit Röthel EuR 2001, 908 (913). 
(Frankreich bzw. Österreich) bezogen waren. Gerade die Gegenüberstellung der Entscheidungen Spanische Erdbeeren bzw. Schmidberger einerseits und Fra.bo andererseits, macht zweitens deutlich, dass die Institute der mittelbaren und der unmittelbaren Drittwirkung unterschiedliche Adressaten erfassen. Die Bindung (Sanktionierung) des einen (Staat) steht gegenüber der Verpflichtung des anderen (Privater) nicht in einem logischen Ausschlussverhältnis ${ }^{109}$. Vielmehr spricht drittens die effektive Durchsetzung der Grundfreiheiten dafür, sowohl die Schutzpflichtenkonstruktion als auch die Figur der unmittelbaren Drittwirkung kumulativ im Rahmen jeder einzelnen Grundfreiheit als Instrument anzuerkennen.

Im konkreten Einzelfall kommt es darauf an, auf welche Weise dem Schutz und der Förderung des Binnenmarktes am effektivsten Rechnung getragen werden kann ${ }^{110}$. Es kann weder pauschal von der primären Anwendung der Schutzpflichtenkonstruktion noch von einer generell höheren Effektivität der direkten Privatrechtsbindung ausgegangen werden $^{111}$. Theoretisch erscheint es sogar denkbar, dass im konkreten Fall beide Figuren Anwendung finden.

\section{Rechtfertigung von privatem Handeln}

Steht im Lichte der vorstehenden Ausführungen fest, dass Privatrechtssubjekte in weitem Umfang an die Grundfreiheiten gebunden sind, stellt sich die in der EuGH-Judikatur gleichfalls noch nicht abschließend geklärte Folgefrage nach den Rechtfertigungsmöglichkeiten. Parallel zum staatlichen Handeln kann insoweit zwischen geschriebenen und ungeschriebenen Rechtfertigungsgründen unterschieden werden. Im einen wie im anderen Fall ist zudem eine Verhältnismäßigkeitsprüfung durchzuführen ${ }^{112}$.

Paradigmatisch für die prinzipielle Möglichkeit einer Anwendung der geschriebenen und ungeschriebenen Rechtfertigungsgründe auf privates Handeln ist die Rs. Bosman. Dort kam der EuGH zunächst bezüglich der ordre publicGründe aus Art. 45 III AEUV zu dem Schluss, »(...) daß die Rechtfertigungsgründe (...) von Privatpersonen geltend gemacht werden [können] «. Der öffentliche oder private Charakter der betreffenden Regelung habe »keinen Einfluss auf die Tragweite oder den Inhalt dieser Rechtfertigungs-

109 So auch Burgi EWS 1999, 327 (330 Rn 46).

110 Ähnlich Röthel EuR 2001, 908 (914).

111 AA Körber (Fn 2), S 807; Streinz/Leible EuZW 2000, 459 (466).

112 Im Grundsatz auch Forsthoff EWS 2000, 389 (395f). gründe ${ }^{113}$. Darüber hinaus stellte der Gerichtshof, mit Blick auf die nicht-diskriminierenden Transferregelungen, explizit die Frage nach dem Vorliegen von »zwingenden Gründen des Allgemeininteresses «114. In der späteren Entscheidung Viking hat der Gerichtshof den von FSU/ITF verfolgten "Schutz der Arbeitnehmer« sogar explizit zu den zwingenden Gründen des Allgemeininteresses gezählt ${ }^{115}$. Für dieses Vorgehen spricht eine Kontrollüberlegung: Begründet man die Privatrechtswirkung (zumindest auch) damit, dass der Staat seiner Grundfreiheitenbindung nicht dadurch entfliehen kann, dass er Regelungsbefugnisse auf Private verlagert, dann müssen die gebundenen Privaten umgekehrt (gewissermaßen kompensatorisch) grundsätzlich zumindest diejenigen Rechtfertigungsgründe geltend machen können, die auch dem Staat zu Gebote gestanden hätten. $\mathrm{Zu}$ prüfen ist mit anderen Worten, ob bei einem hypothetischen (»gedachten«) Staatshandeln eine Rechtfertigung möglich gewesen wäre.

Dessen ungeachtet bleibt festzuhalten, dass Private typischerweise keine Gemeinwohlinteressen verfolgen, sondern privatnützig handeln. Würde man die Rechtfertigungsmöglichkeit nun im Horizontalverhältnis auf die geschriebenen Gründe und die zwingenden Erfordernisse des Allgemeininteresses begrenzen, wäre der Rechtfertigungsrahmen denkbar eng gesteckt. Vor diesem Hintergrund sind zwei Lösungsansätze zur Entwicklung passgenauer Rechtfertigungsgründe denkbar.

In der Rs. Angonese hat der Gerichtshof zunächst den Weg hin zu einer Anpassung und Erweiterung der ungeschriebenen Rechtfertigungsgründe auf Drittwirkungskonstellationen gewiesen ${ }^{116}$. Gefordert werden nicht mehr »zwingende Gründe des Allgemeininteresses« sondern lediglich »sachliche Erwägungen«. Diese müssen dann »(...) unabhängig von der Staatsangehörigkeit der betroffenen Personen und in Bezug auf das berechtigterweise verfolgte Ziel verhältnismäßig [sein] «"117. Eine Rückbindung an Gemeinwohlbelange wird nicht vorausgesetzt. Da im vorliegenden Urteil jedoch eine (versteckte) Diskriminierung aus Gründen der Staatsangehörigkeit vorlag, wurden »sachliche Erwägungen« durch den Gerichtshof nicht ge-

113 EuGH, Rs C-415/93, Slg 1995, I-4921 Rn 86 - Bosman; zustimmend Forsthoff EWS 2000, 389 (395); kritisch Streinz/Leible EuZW 2000, 459 (461).

114 EuGH, Rs C-415/93, Slg 1995, I-4921 Rn 104 - Bosman; s auch GA Lenz, Schlussanträge v 20. 09. 1995, Rs C-415/93, Slg 1995, I-4921 Rn 216 - Bosman, »überragend wichtiges Verbandsinteresse« als zwingender Grund des Allgemeininteresses.

115 EuGH, Rs C-438/05, Slg 2007, I-10779 Rn 75 ff - Viking.

116 Forsthoff EWS 2000, 389 (395).

117 EuGH, Rs C-281/98, Slg 2000, I-4139 Rn 42 - Angonese. 
prüft ${ }^{118}$. Der EuGH hat diese Formulierung in seiner späteren Judikatur freilich nicht wieder aufgegriffen ${ }^{119}$. Ein Grund hierfür mag sein, dass es dem Begriff der »sachlichen Erwägungen« an jeglicher dogmatischen Verwurzelung und Abgrenzungsschärfe fehlt ${ }^{120}$.

Vorzugswürdig erscheint vor diesem Hintergrund vielmehr die direkte Heranziehung der EU-Grundrechte als Rechtfertigungsgründe ${ }^{121}$. Hiermit korrespondiert es, wenn auch der EuGH in den Rs. Bosman, Viking und Laval anerkennt, dass sich ein privater Verpflichteter innerhalb einer Drittwirkung der Grundfreiheiten zur Rechtfertigung auf Unionsgrundrechte, wie die Versammlungs- und Vereinigungsfreiheit (Art. $12 \mathrm{GRCh})^{122}$ sowie das Recht auf Kollektivverhandlungen und Kollektivmaßnahmen (Art. 28 GRCh) ${ }^{123}$ berufen kann.

Dieser Ansatz hat zwar zur Folge, dass in allen Fällen einer Grundfreiheitenbindung Privater ein schonender Ausgleich mit den kollidierenden Grundrechten des Verpflichteten vorzunehmen ist. Hiermit wird aber gerade dem Umstand Rechnung getragen, dass die Anerkennung einer Drittwirkung der Grundfreiheiten bereits per definitionem eine Gefährdung zumindest der grundrechtlich geschützten Privatautonomie des Verpflichtungsadressaten bedeutet.

Der Konflikt zwischen Grundfreiheiten und Grundrechten, als prinzipiell gleichrangigen Garantien, muss dann im Wege eines schonenden Ausgleichs, unter möglichst optimaler Realisierung der widerstreitenden Rechte aufgelöst werden ${ }^{124}$. Gefordert ist mithin die Herstellung praktischer Konkordanz durch »verhältnismäßige« Zuordnung ${ }^{125}$. Modellhaft hierfür steht - im Kontext der mittel-

118 EuGH aaO, Rn 45 iVm. 42; allg zur - umstrittenen und in der EuGH-Judikatur uneinheitlich beurteilten - Anwendbarkeit der ungeschriebenen Rechtfertigungsgründe auf diskriminierende Maßnahmen Dauses/Brigola Dauses (Hrsg) EU-Wirtschaftsrecht, EL 2013, C.I. Rn $275 \mathrm{ff}$.

119 EuGH, Rs C-94/07, Slg 2008, I-5939 - Raccanelli; EuGH, Rs C172/11 NZA 2012, 863 - Erny.

120 Kritisch auch Ehlers JK 01, EG Art 39/1.

121 Vgl im Kontext staatlichen Handelns EuGH, Rs C-112/00, Slg 2003, I-5659 - Schmidberger.

122 EuGH, Rs C-415/93, Slg 1995, I-4921 Rn 79f - Bosman, wo die Prüfung der Grundrechte unsystematisch bei der Anwendbarkeit der Grundfreiheit erfolgt.

123 EuGH, Rs C-438/05, Slg 2007, I-10779 Rn 77 - Viking; EuGH, Rs C-341/05, Slg 2007, I-11767 Rn 91 - Laval.

124 So auch Löwisch Die horizontale Direktwirkung der Europäischen Grundfreiheiten, 2009, S 263 f; Graber Die unmittelbare Drittwirkung der Grundfreiheiten, 2002, S 226; Ganten (Fn 35), S $176 \mathrm{f}$.

125 Grundlegend zu dieser Rechtsfigur im nationalen Verfassungsrecht $K$. Hesse Grundzüge des Verfassungsrechts der Bundesrepublik Deutschland, 20. Aufl 1995, Rn $317 \mathrm{ff}$; für eine Übertragbarkeit auf die baren Drittwirkung - die Rs. Schmidberger, in der der Gerichtshof einen schonenden Ausgleich zwischen der Warenverkehrsfreiheit einerseits und der Meinungsäußerungs- und Versammlungsfreiheit andererseits hergestellt hat ${ }^{126}$.

\section{Resümee}

Insgesamt bleibt festzuhalten, dass sich die Drittwirkungsdogmatik des Gerichtshofs auf ein logisch schlüssiges System zurückführen lässt. Zwar stellt der Wunsch nach Konvergenz der Grundfreiheiten keine eigenständige Argumentationsfigur dar. Die vorstehende Analyse hat aber deutlich gemacht, dass die in Richtung einer einheitlichen Dogmatik für alle Grundfreiheiten weisende Judikatur auch im Bereich der Drittwirkung hohe Überzeugungskraft entfaltet.

Im Einzelnen ist insoweit ein Fünffaches festzuhalten. Erstens können alle Grundfreiheiten eine unmittelbare Drittwirkung gegenüber Diskriminierungen und Beschränkungen von intermediären Gewalten, unabhängig von einem Handeln im Innen- oder Außenverhältnis, entfalten. Entscheidend kommt es dabei auf die Ausübung einer rechtlichen Autonomie als funktionales Äquivalent zu staatlichem Handeln an (Test des hypothetischen Staatshandelns). Zweitens folgt hieraus zugleich die grundsätzliche Absage an eine Bindung sonstiger (»einfacher") Privater an die Grundfreiheiten. Eine Ausnahme kann allenfalls für den durch das Über- und Unterordnungsverhältnis geprägten Sonderfall der Arbeitnehmerfreizügigkeit gelten. Im Übrigen trifft »einfache« Private aus Gründen des Schutzes der Privatautonomie »nur« eine mittelbare Drittwirkung in Form der Schutzpflichtenkonstruktion. Das Institut der Schutzpflicht des Staates ist keine Besonderheit der Warenverkehrsfreiheit, sondern kann drittens auch auf alle anderen Grundfreiheiten übertragen werden. Aus dem soeben Erwähnten folgt wiederum viertens die Möglichkeit, dass die Grundfreiheiten sowohl unmittelbar als auch mittelbar gegenüber Privaten wirken. Für die Abgrenzung kommt es entscheidend darauf an, welcher Lösungsansatz im Einzelfall zu einer effektiveren Verwirklichung des Unionsrechts führt (effet utile). Als Konsequenz der umfänglichen Bindung Privater an die Grundfreiheiten ergibt sich schließlich fünftens ebenfalls eine Erweiterung der Rechtfertigungsmöglichkeiten. Zum einen können sich Private

Grundfreiheitendogmatik Jarass EuR 2013, 29 (30f); kritisch Röthel EuR 2001, 908 (916).

126 EuGH, Rs C-112/00, Slg 2003, I-5659 Rn 65 ff, 95 - Schmidberger; s auch IV.2. 
grundsätzlich auch auf die ursprünglich für hoheitliche Maßnahmen zugeschnittenen Rechtfertigungsgründe berufen (Test des hypothetischen Staatshandelns). Diese umfassen insbesondere die ordre public-Gründe sowie die zwingenden Erfordernisse/Gründe des Allgemeininteres- ses. Zum anderen spielen im Kontext der unmittelbaren Drittwirkung die EU-Grundrechte eine besondere Rolle, da zumindest die grundrechtlich geschützte Privatautonomie regelmäßig in den Abwägungsprozess eingebracht werden kann. 